\title{
Is specialist centre delivery of gastroschisis beneficial?
}

\author{
G Nicholls, V Upadhyaya, P Gornall, R G Buick, J J Corkery
}

\begin{abstract}
This study aims to establish the usefulness of delivering neonates with gastroschisis in a regional obstetric and neonatal centre without the facility of on site surgery.

A retrospective analysis was performed on the notes of 43 consecutive neonates with gastroschisis referred to Birmingham Children's Hospital over a 10 year period. Two groups were compared: those delivered at the regional obstetric centre $(n=9)$ and those delivered peripherally $(n=34)$. Both groups underwent postnatal transfer.

There were no significant differences with regard to gestational age, birth weight, caesarean section rate, time to operation, and mortality. Primary closure rates were $89 \%$ for the regional centre group and $94 \%$ for the peripheral hospital group. Mean time to full enteral feeding was 24 days for the regional centre group and 23 days for those delivered peripherally.
\end{abstract}

These data show that good results can be achieved with postnatal transfer. If on site surgery is not available, neonatal services are adequate peripherally, and the transfer distance is not too great, then delivery in a regional obstetric centre with subsequent postnatal transfer offers no advantage.

(Arch Dis Child 1993; 69: 71-73)

Gastroschisis continues to be one of the rarer neonatal emergencies occurring one in every 10000 births, although there is some evidence that this incidence has increased over the last 10 years in Europe and the United States. ${ }^{1}$ It was a universally fatal condition until 1948 when Watkins described the first surgical repair. Due to improved neonatal care, the mortality has fallen steadily and is now low and of the order of $4-10 \% .^{23}$ Interest now centres around providing a management strategy that reduces morbidity and time to full intestinal recovery to a minimum. Particular attention has focused on the type of closure employed. Also, since the advent of antenatal diagnosis, controversy has arisen over the optimal timing and route of delivery. It has been suggested that delivery in a specialist obstetric centre with neonatal surgery on site, facilitating early operation, leads to a shorter postoperative course. ${ }^{3}$ More recently it has been stated that antenatal diagnosis, elective caesarean section at 37 to 38 weeks' gestation in a specialist obstetric centre, and immediate primary repair confer an advantage. ${ }^{4}$

It is not clear, however, whether the practice of transferring neonates in utero with an antenatal diagnosis of gastroschisis to a regional obstetric centre without on site surgery, offers an advantage. Clearly, postnatal transfer to the surgical unit will still be required with its associated maternal anxiety and additional expense.

This paper examines the experience at Birmingham Children's Hospital of dealing with postnatal transfers of gastroschisis from the regional obstetric centre and peripheral hospitals over a 10 year period.

\section{Methods}

A retrospective analysis was performed on the notes of 43 consecutive neonates with gastroschisis admitted to Birmingham Children's Hospital over a 10 year period: 1 January 1980-31 December 1989. These all consisted of postnatal transfers, either from the regional obstetric centre $(n=9)$ or from peripheral hospitals $(n=34)$. The distance did not exceed 90 kilometres for the peripheral hospital transfers. The regional obstetric centre is 4 kilometres away from the surgical centre.

The two groups were compared with respect to gestational age, birth weight, caesarean section rate, time to operation, and mortality. Primary closure, defined as skin and muscle closure, was achieved whenever possible at the discretion of the operating surgeon.

Time to full enteral feeding was taken as the initial outcome measure. Those patients who died and those with the complication of intestinal atresia were excluded from this part of the analysis (see table 2).

Normally distributed data were analysed statistically using the unpaired $t$ test and other data using the Mann-Whitney U Test. The relationship between place of delivery and initial mortality was assessed using the $\chi^{2}$ test.

\section{Results}

The comparison between the two groups is summarised in table 1 .

Eight of the nine neonates from the regional centre were diagnosed on ultrasound scan antenatally $(89 \%)$ compared with nine of 34 $(26 \%)$ for the peripheral hospital group. Caesarean section rates were comparable. All sections were for reasons other than gastroschisis.

A $93 \%$ primary closure rate was achieved. One neonate from each group required a Silastic pouch. A further neonate from the peripheral group underwent initial skin closure only. Overall mortality was $7 \%$. This consisted of three deaths in the peripheral hospital 
Table 1 Comparison of data for neonates with gastroschisis transferred postnatally from regional obstetric centre or peripheral hospitals

\begin{tabular}{lccc} 
& $\begin{array}{l}\text { Regional obstetric } \\
\text { centre } \\
(n=9)\end{array}$ & $\begin{array}{l}\text { Peripheral } \\
\text { hospital } \\
(n=34)\end{array}$ & $p$ Value \\
\hline Mean (SD) gestation (weeks) & $37(1.45)$ & $37 \cdot 5(2 \cdot 0 \dot{u})$ & $>0 \cdot 1^{\star}$ \\
Mean (SD) birth weight (g) & $2690(500)$ & $2500(500)$ & $>0 \cdot 1^{\star}$ \\
No (\%) caesarean sections & $1(11)$ & $5(15)$ & $>\cdot 1 \dagger$ \\
Mortality & 0 & $3(9)$ & $>0 \cdot 5 \dagger$ \\
No (\%) primary closures & $8(89)$ & $32(94)$ & $>0 \cdot 1 \neq$ \\
Mean time (hours) to operation & $4 \cdot 5$ & $5 \cdot 9$ & $>0 \cdot 1 \neq$ \\
Mean time (days) to full enteral feeding & 24 & 23 & \\
\hline
\end{tabular}

$\star$ Unpaired $t$ test, $\dagger \chi^{2}$ test, $¥ M a n n-$ Whitney $U$ test.

Table 2 Patients with atresias: treatment and time to full enteral feeding

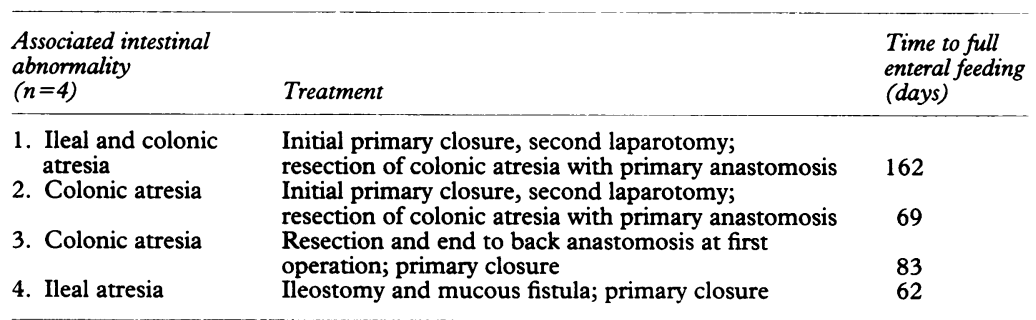

transfer groups. This did not reach statistical significance. All three deaths occurred before 1983 and were undiagnosed antenatally.

The first death occurred as a result of antepartum haemorrhage that occurred on the day of delivery at 34 weeks' gestation. Delivery was by emergency caesarean section. The infant was in very poor condition at birth and after resuscitation primary closure was performed at 11 hours of age. The bowel was noted to be in good condition. Death occurred at 17 hours of age after increasing hypotension, acidosis, and renal failure.

The second death occurred in a patient with almost total small bowel necrosis at 3 hours of age. This was almost certainly necrotic at delivery. All the necrotic bowel was resected leaving only $10 \mathrm{~cm}$ of small bowel. This patient died at 5 days of age.

The third death occurred in a neonate noted to have a necrotic ascending colon, twisted mesentery with a single supplying vessel, and a small defect of approximately $0.5 \mathrm{~cm}$. He arrived at the surgical centre at 2.5 hours of age, at which time the bowel was untwisted, and operation was performed immediately. The remaining bowel was severely compromised and did not improve on enlarging the defect. The necrotic ascending colon was resected with primary anastamosis and primary closure was achieved without tension. A second look laparotomy was performed at 48 hours because of a declining clinical condition and all the remaining bowel was seen to be necrotic.

Time to full enteral feeding was 24 days (range 10-14 days) for those transferred from the regional obstetric centre and 23 days (range 11-51 days) for those transferred from peripheral hospitals. This difference was not significant. The analysis of time to full enteral feeding excludes four patients, all born in peripheral hospitals, who were found to have intestinal atresias at operation; all survived. The site of atresia, operative management, and time to full enteral feeding are shown in table 2 .

\section{Discussion}

The current management of gastroschisis results in a low mortality. The overall mortality in this series (7\%) compares favourably with that quoted by others. ${ }^{235}$ Although statistical significance was not reached, it is noteworthy that all three deaths occurred in the peripheral hospital transfer group. The first death was due to factors unrelated to gastroschisis. In the second case with total small bowel necrosis present at birth, it is unlikely that delivery in the regional obstetric centre would have affected the outcome. This would also apply if on site neonatal surgery had been available. In the third case, noted to have a twisted mesentery, immediate untwisting by an on site surgeon with immediate operation may have salvaged the bowel.

Primary closure rates of $89 \%$ for regional centre and $94 \%$ for peripheral hospital groups are also very respectable. It is still controversial as to whether primary closure offers a better outcome. However, it is generally accepted that staged procedures have more complications in terms of sepsis, bowel infarction, and perforation and separation of protheses. ${ }^{6-8}$

Stringer $e t$ al found a time to full enteral feeding of 29.9 days for those neonates with gastroschisis delivered at a regional centre possessing neonatal surgery on site. ${ }^{3}$ The neonates in our series transferred from peripheral hospitals had a mean time to full enteral feeding of 23 days. Delivery at the regional centre with postnatal transfer did not shorten this time.

The data presented in this paper demonstrate that good results can be achieved in neonates with gastroschisis undergoing postnatal transfer. There were no significant differences between the regional centre and peripheral hospital groups.

If on site surgery is not available, neonatal se:vices are adequate peripherally and the transfer distance is not too great, then our results suggest that delivery in a regional obstetric and neonatal centre offers no advantage. Only one patient out of 43 may have been helped by delivery at a regional centre with surgery available on site.

In utero transfer is a very disrupting process for the family involved. Before advocating such a policy as a routine the full benefits of such transfer need to be assessed. We believe that our observations tend to favour the argument against transfer.

1 Torfs C, Curry C, Roeper P. Gastroschisis. F Pediatr 1990; 116: $1-6$

2 Crabbe DCG, Thomas DFM, Beck JM, Spicer RD. Prenatally diagnosed gastroschisis: a case for preterm delivery? Pediatric Surgery International 1991; 6: 108-10.

3 Stringer MD, Brereton RJ, Wright VM. Controversies in the management of gastroschisis: a study of $\mathbf{4 0}$ patients. Arch Dis Child 1992; 66: 34-6.

4 Swift RI, Singh MP, Ziderman DA, Silverman M, Elder MA, Elder MG. A new regime in the management of gastroschisis. F Pediatr Surg 1992; 27: 61-3.

5 Caniano DA, Brokaw B, Ginn-Pease ME. An individualised approach to the management of gastroschisis. $f$ Pediatr Surg 1990; 25: 297-300.

6 Filston MC. Gastroschisis - primary fascial closure: the goal for optimal management. Ann Surg 1983; 197: 260-4.

7 Ein SH, Rubin SZ. Gastroschisis: primary closure or silon pouch? ₹ Pediatr Surg 1980; 15: 549-52.

8 Canty TG, Collins DL. Primary fascial closure in infants with gastroschisis and omphalocele: a superior approach. f Pediatr Surg 1983; 18: 707-12. 